\title{
Alterations in Neural Responses and Pain Perception in Older Adults During Distraction
}

\author{
Ana María González-Roldán, PhD, Juan Lorenzo Terrasa, PhD, Carolina Sitges, PhD, \\ Marian van der Meulen, PhD, Fernand Anton, PhD, and Pedro Montoya, PhD
}

\begin{abstract}
Objective: Although it is acknowledged that pain may be modulated by cognitive factors, little is known about the effect of aging on these control processes. The present study investigated electroencephalographical correlates of pain processing and its cognitive modulation in healthy older individuals.

Methods: For this purpose, the impact of distraction on pain was evaluated in 21 young (9 men; 20.71 [2.30]) and 20 older (10 men; 66.80 [4.14]) adults. Participants received individually adjusted electrical pain stimuli in a high-distraction condition (one-back task) and in a low-distraction condition (simple letter response task). Pain-related evoked potentials and pain ratings were analyzed.

Results: Both groups rated pain as less intense $(F(1,39)=13.954, p<.001)$ and less unpleasant $(F(1,39)=10.111, p=.003)$ when it was experienced during the high- rather than the low-distraction condition. However, in comparison to younger participants, older adults gave higher unpleasantness ratings to painful stimulation $(F(1,39)=4.233, p=.046)$, accompanied by attenuated neural responses $(\mathrm{N} 1-\mathrm{P} 1$ and P3 amplitudes), regardless of the distraction condition $(F(1,38)=6.028[p=.019]$ and $F(1,38)=6.669[p=.014]$, respectively).

Conclusions: Older participants felt pain relief through distraction, like younger participants. However, we also found that aging may enhance affective aspects of pain perception. Finally, our results show that aging is characterized by reduced neural processing of painful stimuli. This phenomenon could be related to the increased vulnerability of older participants to develop chronic pain.
\end{abstract}

Key words: pain, aging, cognitive modulation, distraction, electroencephalography.

\section{INTRODUCTION}

C urrent demographic trends require a deepening of knowledge on age effects on pain processing to promote a functional and healthy aging population. In a recent meta-analysis (1), the authors concluded that aging seems to be associated with increased pain thresholds. However, changes in pain tolerance were not consistent across studies, and studies addressing the neural mechanisms underlying pain perception and modulation were too scarce to be considered.

Aging affects the organization and functioning of pain-related neural networks (2). In this sense, it has been suggested that the increased prevalence of chronic pain in older adults (reaching $>50 \%$ ) could be associated with age-related changes in pain inhibition (1-3). The frontal cortex network has been shown to be a key region in bottom-up (4) and top-down pain modulations triggered by psychological factors $(5,6)$. Given that the frontal cortex is one of the most vulnerable brain regions in aging (2), cognitive pain modulation is likely to be affected in this population. In agreement, Zhou and colleagues (7) showed that, in healthy older adults, poorer executive control was related to lower pain tolerance in the cold pressor test. Moreover, it has been shown that distraction from pain was less efficient in older persons (8). However, these studies were based on self-reports of pain intensity, and to date, no research has directly analyzed electroencephalographical (EEG) correlates of cognitive pain modulation in older individuals.

The present study aimed to investigate the neural processing of pain and the efficacy of cognitive modulation via distraction in older healthy participants in comparison to younger participants. For this purpose, pain-evoked potentials were recorded while participants received painful electrical stimuli concomitantly to the execution of a high-distraction (one-back) and a low-distraction (simple letter response) task. Electrically induced pain-evoked potentials are characterized by a negative peak at 100 to 150 milliseconds (N1) and a subsequent positive peak at 200 to 250 milliseconds (P1), with maximal peak amplitude around the vertex $(9,10)$. Moreover, later components such as P3 are also elicited (11). Importantly, these potentials are not a mere reflection of sensory pain processing but are highly influenced by bottom up and top-down mechanisms such as attentional selection and stimulus salience $(12,13)$. Because aging is associated with a decline in attentional control $(14,15)$, we expect that distraction will be less efficient as a cognitive modulator of pain in older persons, who will therefore show altered N1-P1 complex and

ANOVA $=$ analysis of variance, $\mathbf{C B P T}=$ cluster-based permutation test, $\mathbf{E E G}=$ electroencephalographical, ISI = interstimulus interval, VAS $=$ visual analog scale

\section{SDC Supplemental Content}

From the Cognitive and Affective Neuroscience and Clinical Psychology, Research Institute of Health Sciences (IUNICS) and Balearic Islands Health Research Institute (IdISBa) (González-Roldán, Terrasa, Sitges, Montoya), University of the Balearic Islands (UIB), Palma, Spain; and Institute for Health and Behaviour, FLSHASE/INSIDE (van der Meulen, Anton), University of Luxembourg, Esch-sur-Alzette, Luxembourg.

Address correspondence to Ana María González-Roldán, PhD, Cra. de Valldemossa km 7.5, 07122 Palma, Spain. E-mail: anamaria.gonzalez@uib.es

Received for publication January 15, 2020; revision received August 4, 2020.

DOI: $10.1097 /$ PSY.0000000000000870

Copyright $\odot 2020$ by the American Psychosomatic Society 
P3 amplitudes and higher pain ratings as compared with younger participants, especially in the high-distraction condition.

\section{MATERIALS AND METHODS}

\section{Participants}

Most participants were recruited from the University of the Balearic Islands (the older group was recruited from a senior program of the university). Twenty-three healthy older and 21 younger adults participated in the experiment (see Table 1 for sociodemographic and clinical data). However, three older participants were excluded because they were identified as outliers in the analyses of task performance: two participants presented a percentage of hits of $41 \%$ and $16.22 \%$, respectively, and one participant did not press any button in the low-distraction task (see Table 2 for descriptive data of task performance in each group). Therefore, the final sample was composed of 20 healthy older adults (10 men; 66.80 [4.14] years, age range of 60-79 years) and 21 healthy young adults (9 men; 20.71 [2.30] years, age range of 18-25 years). Data were collected between December of 2017 and January 2018.

All participants were interviewed in a screening session to exclude those presenting with any of the following criteria: any psychiatric or neurological condition, acute or chronic pain, history of drug abuse, cognitive impairment (operationalized as a score $<27$ in the Mini-Mental State Examination (16)), or left handed. In regard to medication, five older participants were medication-free, 5 were taking antihypertensives, and the rest were taking other drugs (i.e., seven participants were taking cholesterol-lowering agents, two antidepressants [despite not showing depressive symptoms in the last 7 years], one metformin, two tamsulosin, four antacids). Four younger participants were taking oral contraceptives. All individuals were naive to the experiment and gave informed consent after the experimental procedure was explained. The study was conducted in accordance with the Declaration of Helsinki (1991) and was approved by the Ethics Committee of the Balearic Islands.

\section{Sociodemographic and Clinical Assessment}

Before the day of the main experiment, all participants underwent an initial a semistructured interview about their medical and psychological history. In addition, the following questionnaires were completed: the Spanish versions of the Mini-Mental State Examination (16), the Patient Health Questionnaire-9 (17), the Generalised Anxiety Disorder Assessment questionnaire (18), the Pain Catastrophizing Scale (19), and the Edinburgh Handedness Inventory (20). Finally, on the same day of the main experiment, the Positive and Negative Affect Schedule (21) was filled out to assess the participant's mood at laboratory arrival.

\section{Painful Stimulation}

Electrical pain stimuli were delivered using a DS7A Digitimer (Digitimer Limited, Welwyn Garden City, United Kingdom). Electrical stimuli of 1-millisecond duration were delivered to the ventral side of the nondominant wrist by using a modified version of the electrode described by Inui and colleagues (22). Because we were especially interested in evaluating the brain processing of pain and its modulation by cognitive factors, we wanted to minimize the risk that a priori differences in sensory thresholds could be responsible for the observed differences between the two age groups. The intensity of pain stimulation during the experiment was hence adapted to each participant's individual pain threshold. Individual sensory and pain thresholds were determined in three ascending series using the method of limits (23) and were computed as the mean of the two last series. The thresholds were determined by presenting pulses of 1 millisecond ranging from $0.5 \mathrm{~mA}$ and increasing in steps of $0.5 \mathrm{~mA}$ with a 5-second interstimulus interval (ISI). Sensory threshold was defined as the minimum stimulation perceived by the participant and pain threshold as the minimum intensity required to be perceived as painful.

\section{Experimental Design}

\section{Experimental Protocol and Distraction Tasks}

We used a pain distraction paradigm, in which painful stimulation was presented to participants while they were performing the oneback task or while they were performing a simple letter response task. The one-back task was set as the high-distraction condition because it is demanding higher executive resources and therefore competing with pain for attention. In addition, it is an easy task

TABLE 1. Participants' age, questionnaire data, and pain thresholds

\begin{tabular}{lccc}
\hline & Younger group $(n=21), M(S D)$ & Older group $(n=20), M(S D)$ & Statistics \\
\hline Age, $y$ & $20.74(2.30)$ & $66.80(4.14)$ & $t(39)=-44.345, p<.001$ \\
Mini-Mental & - & $29.25(0.97)$ & - \\
PHQ-9 & $4.06(3.57)$ & $3.25(3.94)$ & $t(36)=0.657, p=.515$ \\
GAD-7 & $4.83(4.05)$ & $3.40(3.99)$ & $t(36)=1.098, p=.280$ \\
PANAS, positive affect & $47.62(5.35)$ & $46.45(8.98)$ & $t(39)=0.509, p=.613$ \\
PANAS, negative affect & $44.52(5.01)$ & $43.65(9.30)$ & $t(39)=0.372, p=.713$ \\
PCS, rumination & $6.19(3.71)$ & $4.85(4.04)$ & $t(39)=1.107, p=.275$ \\
PCS, magnification & $11.57(6.14)$ & $9.15(6.28)$ & $t(39)=1.248, p=.219$ \\
PCS, helplessness & $15.05(7.75)$ & $12.70(9.07)$ & $t(39)=0.893, p=.378$ \\
Sensory threshold & $0.31(0.23)$ & $0.33(0.16)$ & $F(2,38)=0.050, p=.824$ \\
Pain threshold & $1.08(0.38)$ & $1.73(0.89)$ & $F(2,38)=8.886, p=.005$ \\
\end{tabular}

$\mathrm{M}(\mathrm{SD})$ values are displayed for younger and older participants. Statistical values and significance of group differences are shown.

M (SD) = mean (standard deviation); PHQ-9 = Patient Health Questionnaire; GAD-7 = Generalised Anxiety Disorder Assessment; PANAS = Positive and Negative Affect Schedule; PCS = Pain Catastrophizing Scale. 
equally attainable for young and old adults. To further equate task demand for young and older participants, we adapted the ISI between probes through a calibration procedure, performed before the main experiment (see the Practice and Task Difficulty Calibration section), similarly to Buhle and Wager (24). A simple letter response task was used as a low-distraction condition. This condition requires minimal executive processes but in turn constitutes a good control condition, with presentation of letters and issuing responses in a similar way as the one-back task does.

In the one-back task, a series of letters was displayed in the center of a computer screen and participants were asked to indicate whether each letter was the same as (target) or different (no target) from the previous one. We structured the task in blocks of $60 \mathrm{sec}-$ onds in which approximately $33 \%$ of the letters were targets. Each letter was presented foveally for 840 milliseconds, followed by the ISI (the mean durations of this interval after the calibration procedure were 380.70 [70.60] milliseconds for younger participants and 414.90 [72.08] milliseconds for older participants). Then, participants pressed a red or a green button ("1" and " 2 " keys on a standard numeric keyboard) to indicate responses of either "not the same" or "same." The position of the keys was counterbalanced across participants. Responses could be made any time during the presentation of the letter or during the ISI. Participants were instructed to respond to every letter as quickly as possible. We recorded reaction times and the number of hits, misses, correct rejections, false-positives, and missing responses as a measure of task performance. In the low-distraction condition, we also presented a series of letters, but participants were informed that the letters would never repeat and that their task was simply to respond to each one by pressing one of the two keys (they were instructed to use always the same key).

A total of six blocks were presented for each distraction condition in counterbalanced order. Half of the sample started with the high-distraction condition and the other half with the low-distraction condition. Concomitantly to the distraction tasks, three painful stimuli of 1-millisecond duration were delivered during the 60-second blocks (randomly distributed every 15-20 seconds). Each painful stimulus appeared during the blank ISI at least 1150 milliseconds after the onset of the letter and always after the response was executed. Hence, for all participants, 18 painful stimuli were applied during each condition. After each block, participants rated the average intensity and unpleasantness of the painful stimulation (composed by the three pain stimuli presented in each block) with a computerized visual analog scale (VAS) displayed onto the screen. Scales ranged from no pain/not unpleasant to worst pain imaginable/ highly unpleasant. After the ratings, a 30 -second fixation cross was presented.

\section{Practice and Task Difficulty Calibration}

Before the main experiment, participants were introduced to the pain assessment scales and the distraction tasks. First, participants practiced the one-back task during 26 probes (eight targets). They repeated the practice task as often as needed until all of them understood the task. After the practice task, a calibration procedure of the one-back task similar to that described by Buhle and Wager (24) was performed to ensure the same difficulty level for all participants. For this purpose, initial ISI duration was set to 1850 milliseconds but changed every 29 stimuli according to a target sensitivity assessment evaluated with the nonparametric signal detection measure $A(25)$. This provides a measure of performance accuracy independent of response bias (the tendency to report "yes" or "no" systematically). The ISI duration was adjusted according to the following rules: a) if sensitivity was higher than the targeted level of $A=0.85$, the duration was reduced by 200 milliseconds $*(A-0.85) * 4$; b) if sensitivity was equal or lower than $A=0.85$, the duration was increased by 200 milliseconds * $(A-0.85) *-4 / 3$. Additional adjustments were made until 145 stimuli were completed, yielding five adjustments for each participant. The minimum ISI duration allowed was 300 milliseconds.

After the calibration procedure, participants were asked to practice the low-distraction condition and the VAS rating procedure by presenting a block of 20-letter stimuli in which participants received one painful stimulation in the middle of the block.

\section{Brain Activity Acquisition and Analysis}

EEG signals were recorded using a QuickAmp amplifier (BrainProducts $\mathrm{GmbH}$, Munich, Germany) from 60 scalp electrodes placed according to the international 10/20 system. Electrode signals were recorded using an average reference calculated by the amplifier. An electrooculogram channel was obtained by placing one electrode above and another below the left eye. Electrode impedance was kept below $10 \mathrm{~K} \Omega$. Signals were recorded with a sampling rate of $1000 \mathrm{~Hz}$ using BrainVision Recorder software (BrainProducts $\mathrm{GmbH}$ ).

EEG recordings were further processed offline using BrainVision Analyzer (BrainProducts $\mathrm{GmbH}$ ). A frequency band-pass filter of 0.1 to $0.30 \mathrm{~Hz}$ was applied. For analyses of evoked potentials elicited by electrical painful stimuli, EEG waveforms were segmented in epochs of 1100-millisecond duration (-100 to 1000 milliseconds relative to stimulus onset) and baseline corrected (from -100 to 0 milliseconds). Eye movement artifacts were corrected by using the Gratton and Coles algorithm (26). Thereafter, an artifact rejection protocol with the following criteria was applied: maximal allowed voltage step/ sampling point $=100 \mathrm{mV}$, minimal allowed amplitude $=-100 \mathrm{mV}$, maximal allowed amplitude $=100 \mathrm{mV}$, and maximal allowed absolute difference in the epoch $=100 \mathrm{mV}$. One older participant was excluded from the analyses because of excessive artifact rejection $(50 \%$ rejected trials in each channel). Afterward, EEG waveforms elicited by the painful stimuli were averaged separately for the high- and the low-distraction condition.

Then, two types of analyses were conducted. First, nonparametric cluster-based permutation test (CBPT) was performed by using Fieldtrip toolbox (27) running in MATLAB R2018b. This method allows for testing group differences in high-dimensional neural data, whereas it deals with the multiple comparisons problem (28). Therefore, we used the 60 scalp electrodes recorded and a time window from 0 to 500 milliseconds after the painful stimulus presentation for CBPT. For every sample (electrode $\times$ millisecond), older and younger groups were compared in each distraction condition by means of an independent-samples $t$ test. In addition, high- and low-distraction conditions were compared separately for each group by means of a dependent-samples $t$ test. Samples with $t$ values higher than the critical level $(p<.05)$ were selected and clustered by temporal and spatial adjacency. Next, $t$ values within every cluster were summed to calculate the cluster-level statistics. These observed cluster-level statistics 
were evaluated through a nonparametric permutation test. The permutations were created by randomly assigning labels and running the test 1000 times, retrieving the maximum cluster statistic every time. Only if the observed cluster-level statistics from the real data were larger than $95 \%$ of the maximum cluster statistics in the permutation distribution (Monte-Carlo significance probability), the observed effects were considered as significant.

Because CBPT does not allow to make peak-to-peak subtraction, a second type of analysis was conducted. Based on previous studies on the processing of electrical pain stimuli $(9,10)$, peak-to-peak N1-P1 amplitudes were examined. N1-P1 amplitudes are maximal around the vertex and present a high test-retest reliability $(9,10)$. Taking all this into account, together with visual inspection of our data, peak amplitudes of N1 (negative peak at 100-170 milliseconds) and P1 (positive peak at 160-220 milliseconds) were determined by using a global maxima detection method in $\mathrm{FCz}$ and $\mathrm{Cz}$ electrodes. Then peak-to-peak N1-P1 was computed by calculating the difference between N1 and P1 amplitudes. Thereafter, repeated-measures analyses of variance (ANOVAs) on N1-P1 amplitudes were performed using "group" (young versus older) as a between-subject factor, as well as "electrode" (FCz, Cz) and "task condition" (high versus low distraction) as within-subject factors. Moreover, given that differences yielded by CBPT results (see discussion hereinafter) occurred in a time window and topography matching the well-known posterior P3 potential (11), we decided to quantify P3 to further support our results. For this purpose, we first determined the mean amplitudes from 300 to 500 milliseconds at $\mathrm{Cz}$ and $\mathrm{CPz}$ locations (29-33) and then used these data to execute an ANOVA with the factors "group" (young versus older), "electrode" $(\mathrm{Cz}, \mathrm{CPz})$, and "task condition" (high versus low distraction). Greenhouse-Geisser adjustments were applied, and post hoc Bonferroni-corrected two-tailed paired tests were used. In a prior analysis, we also used "sex" as a between-subject factor, but it was discarded for all the analyses given that we did not find significant effects involving this variable and groups were balanced.

In addition, those amplitudes showing significant differences between groups were correlated with intensity and unpleasantness pain ratings to study the possible relationship between brain activity and subjective pain. These Pearson correlations were computed separately for each group. After Bonferroni correction, the new $p$ value was set at .025 .

Finally, to further assure reliability of our results, we estimated internal consistency of our data. The estimation of internal consistency is crucial in defining how many epochs should be averaged to yield reliable results $(34,35)$. For this purpose, we first analyzed the values of N1-P1 and P3 amplitudes across increasing numbers of epochs $(6,10,12,14,18)$ selected randomly from a same condition. Then, we have tested the internal reliability of the EventRelated Potentials components using the Cronbach $\alpha$ method $(34,35)$, by treating the amplitudes at $\mathrm{FCz}$ and $\mathrm{Cz}$ (for N1-P1) and at $\mathrm{Cz}$ and $\mathrm{CPz}$ (for $\mathrm{P} 3$ ) for the two conditions tasks (high and low distraction) as items (four items) (34). $\alpha$ Values were obtained for each number of epochs. Results showed that N1-P1 amplitude measurements yielded the recommended ERP reliability of 0.70 (36) from six-epoch average (number of epochs: $6=0.882$, $10=0.899,12=0.893)$ and reached an excellent internal consistency from 14 trials onward $(14=0.927,18=0.929)$. ERP reliability was also higher than 0.80 for $\mathrm{P} 3$ amplitudes (number of epochs: $6=0.802,10=0.795,12=0.845,14=0.867,18=0.828)$.

\section{Statistical Analyses of Behavioral and Questionnaire Data}

Group differences on sociodemographic data, self-reports, ISI used during the task, and task performance (hits, misses, correct rejections, false alarms, missing responses, and reaction times) were analyzed with Student $t$ tests. Given that previous research has shown that hypertension (37) and antidepressants (38) can change pain thresholds, electrical sensory and pain thresholds were analyzed with an ANOVA using the presence of hypertension $(n=5)$ and antidepressants intake $(n=2)$ as a covariate. Intensity and unpleasantness VAS ratings were analyzed by repeated-measures ANOVA using "group" (young versus older) as a between-subject factor and task condition (high versus low distraction) as a within-subject factor.

\section{RESULTS}

\section{Sociodemographic and Clinical Data}

Table 1 displays sociodemographic and clinical data, and sensory and pain thresholds of both groups. There were no group differences in the Patient Health Questionnaire-9, Generalised Anxiety Disorder7, and Positive and Negative Affect Schedule, catastrophizing to pain as assessed by the Pain Catastrophizing Scale, and in sensory thresholds to electrical stimulation (all $p$ values $>.05$; Table 1 ). However, pain thresholds were significantly higher in the older group in comparison to the younger one $(F(2,38)=8.886, p=.005)$. No main effect was found in relation to the hypertension and antidepressant covariates $(p=.536)$.

\section{Task Performance}

No significant differences between groups were found in the ISI of the one-back task following the calibration procedure (see Table 2 for $t$ values and descriptive statistics). In addition, there were no group differences for the number of hits, misses, correct rejections, false alarms, and missing responses in the one-back task during the distraction paradigm. However, reaction times to both targets and nontargets were slower in the older group in comparison to the younger group.

\section{Pain Intensity and Unpleasantness Ratings}

The repeated-measures ANOVA of the VAS ratings exhibited significant group differences in unpleasantness ratings $(F(1,39)=$ $4.233, p=.046$ ), showing that older participants rated pain stimuli as more unpleasant compared with young participants (see Table 3 for means and standard deviations). In addition, significant effects of condition were found for intensity $(F(1,39)=13.954, p<.001)$ and unpleasantness ratings $(F(1,39)=10.111, p=.003)$, showing that all participants rated pain as more intense and unpleasant during the low- than during the high-distraction condition. No significant group by condition interaction was found (all $p$ values $>.05$ ).

\section{CBPT Results}

The CBPT revealed that there was a significant difference between the older and younger groups in the high-distraction condition. Although caution must be taken when making inferences about the effect of latency or location with CBPT (39), a cluster in the observed data extended from approximately 280 to 500 milliseconds over central, centroparietal, parietal, and parietoccipital electrodes and mostly in the right hemisphere (Figure 1). In this cluster, painevoked potential amplitude was significantly lower in the older 
TABLE 2. Participants' task performance

\begin{tabular}{lccc}
\hline & Younger group $(n=21), \mathrm{M}(\mathrm{SD})$ & Older group $(n=20), \mathrm{M}(\mathrm{SD})$ & $t$ \\
\hline Interstimulus interval & $380.70(70.60)$ & $414.90(72.08)$ & $t(39)=-1.535, p=.133$ \\
$\%$ Hits & $90.52(5.94)$ & $86.57(13.00)$ & $t(39)=1.262, p=.214$ \\
$\%$ Misses & $8.90(5.57)$ & $11.27(8.64)$ & $t(39)=-1.050, p=.300$ \\
$\%$ False alarms & $0.85(0.74)$ & $1.66(2.05)$ & $t(39)=-1.672, p=.108$ \\
$\%$ Correct rejections & $96.62(1.28)$ & $92.77(11.93)$ & $t(39)=1.434, p=.167$ \\
$\%$ Missing response & $2.03(0.76)$ & $4.67(8.98)$ & $t(39)=-1.310, p=.206$ \\
$\%$ Target reaction time & $441.55(56.65)$ & $592.09(82.67)$ & $t(39)=-6.831, p<.001$ \\
$\%$ Nontarget reaction time & $433.12(57.57)$ & $556.52(92.10)$ & $t(39)=-5.172, p<.001$ \\
\hline
\end{tabular}

$\mathrm{M}(\mathrm{SD})$ and $t$ test results of task performance and pain rating data of the younger and older groups.

$\mathrm{M}(\mathrm{SD})=$ mean (standard deviation).

group when compared with the younger group (Figure 2). In contrast, no significant differences between groups were found in the low-distraction condition or between the two conditions within the younger or the older group.

\section{Peak-to-peak N1-P1 Amplitudes}

A repeated-measures ANOVA showed a significant main effect of group $(F(1,38)=6.028, p=.019)$, revealing that younger participants showed greater N1-P1 amplitudes than did older participants, regardless of the distraction condition (Figure 2). No other significant main effects or interactions were found (all $p$ values $>.05$ ).

\section{Posterior P3 Amplitude}

ANOVA on P3 yielded significant effect of group $(F(1,38)=6,669$, $p=.014)$, showing that young participants exhibited greater amplitudes than did older participants during both the high and low distractions.

\section{Correlation Between Pain-Evoked Potentials and Pain Ratings}

No significant correlations between pain-evoked potential amplitudes and pain ratings were found (all $p$ values $>.05$ ).

\section{DISCUSSION}

The present research aimed to study the effect of aging on cognitive modulation of pain perception. For this purpose, we have analyzed ERPs in response to pain under two different levels of cognitive load when performing a distraction task. Pain rating results suggest that the high-distraction task was able to reduce pain in both older and younger participants. However, we found that older participants displayed increased unpleasantness ratings to pain together with a reduced neural response to painful stimuli

TABLE 3. Intensity and unpleasantness ratings

\begin{tabular}{lcc}
\hline & $\begin{array}{c}\text { Younger group } \\
(n=21), \mathrm{M}(\mathrm{SD})\end{array}$ & $\begin{array}{c}\text { Older group } \\
(n=20), \mathrm{M}(\mathrm{SD})\end{array}$ \\
\hline Low distraction, intensity & $22.52(13.57)$ & $30.78(18.56)$ \\
Low distraction, unpleasantness & $21.13(13.12)$ & $29.67(17.58)$ \\
High distraction, intensity & $18.55(11.62)$ & $27.69(16.29)$ \\
High distraction, unpleasantness & $17.83(10.46)$ & $27.07(14.67)$ \\
\hline
\end{tabular}

$\mathrm{M}(\mathrm{SD})$ values are displayed for younger and older participants.

$\mathrm{M}(\mathrm{SD})=$ mean (standard deviation). in comparison to younger adults, regardless of the distraction condition. The age-related differences cannot be explained by the influence of depression and anxiety, catastrophizing to pain or educational level, because older and younger participants did not differ in these measures.

\section{Analgesic Effects of Distraction}

Although older adults displayed higher overall pain ratings than did young adults, they also benefited from distraction. Indeed, older participants rated the pain as less intense and unpleasant during performance on the one-back task in comparison to the simple response task, to an equal degree as the younger participants. These results contrast with a previous study suggesting that pain modulation induced by a simple cognitive task (tone detection) worsened rather than ameliorated pain in older participants $(7,8)$. Zhou et al. $(7,8)$ argued that this lack of reduction of pain by distraction could be related to reduced functioning of frontal networks. Our results do not contradict the findings by Zhou et al. but extend them by suggesting that a distraction task only aggravates pain in older adults when, together with pain, exhausts their cognitive processing resources. Pain intensity elicited by Zhou et al. (8) was relatively high, whereas the cognitive load of the distraction task was low. This dominance of pain led to an absence of analgesic effect of distraction in the younger group as well as to an increase in perceived pain during distraction in the older group. It is well known that the effectiveness of distraction may decrease under conditions of increased pain intensity or duration (40). Moreover, it seems to be the individual's absorption or involvement in the distracting task rather than the type of task per se, which is positively associated with the efficacy of distraction (40). In our study, the intensity of the pain stimuli was lower, and the high-distraction task, without being too difficult, demanded constant attention leading to a high involvement in the task. We show that, under these conditions, older people can benefit from the effect of distraction. Overall, this result could have important consequences for pain management in an older population because it suggests that, although distraction could be harmful when dealing with intense pain in aging, it could be a promising approach in the case of conditions characterized by less intense pain.

\section{Altered Pain Perception and Related Brain Activity in Older Participants}

We showed a discrepancy between the neural and subjective responses to pain in older participants. Specifically, we found that 

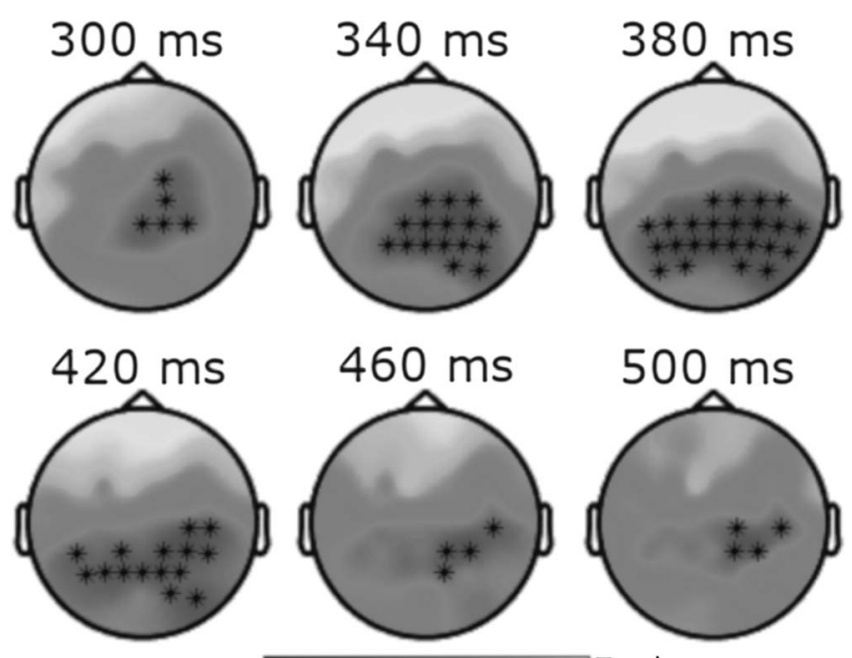

$-5$

0

T-value

FIGURE 1. Results of the cluster-based permutation analysis comparing older and younger under the high-distraction condition. Six representative time points are shown. Asterisks indicate the electrodes where the differences were significant at each time point. Dark gray indicates reduced amplitudes in the older group compared with the younger one. No significant differences were found in the low-distraction condition (not shown).

older participants evaluated painful stimuli as more unpleasant than young participants during both the low- and high-distraction conditions. However, older adults displayed reduced N1-P1 amplitudes under both distraction conditions. In addition, CBPT analyses showed reduced amplitudes in older adults in comparison to younger ones during the high-distraction condition, in a time window (280-500 milliseconds) and topography (centroparietal) matching the well-known P3 potential (11). Simple P3 amplitude quantification analyses support these differences between groups. This age-related reduction in pain-evoked potential amplitudes is in agreement with findings from previous studies using electrical (10), laser (41-43), and thermal (44) painful stimulation. Currently, it is considered that evoked potentials elicited by nociceptive stimuli may represent the activity of a general alarm system related to detect and react to salient

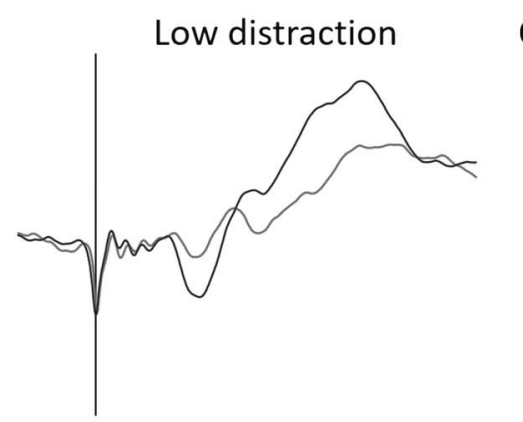

$\mathrm{Cz}$
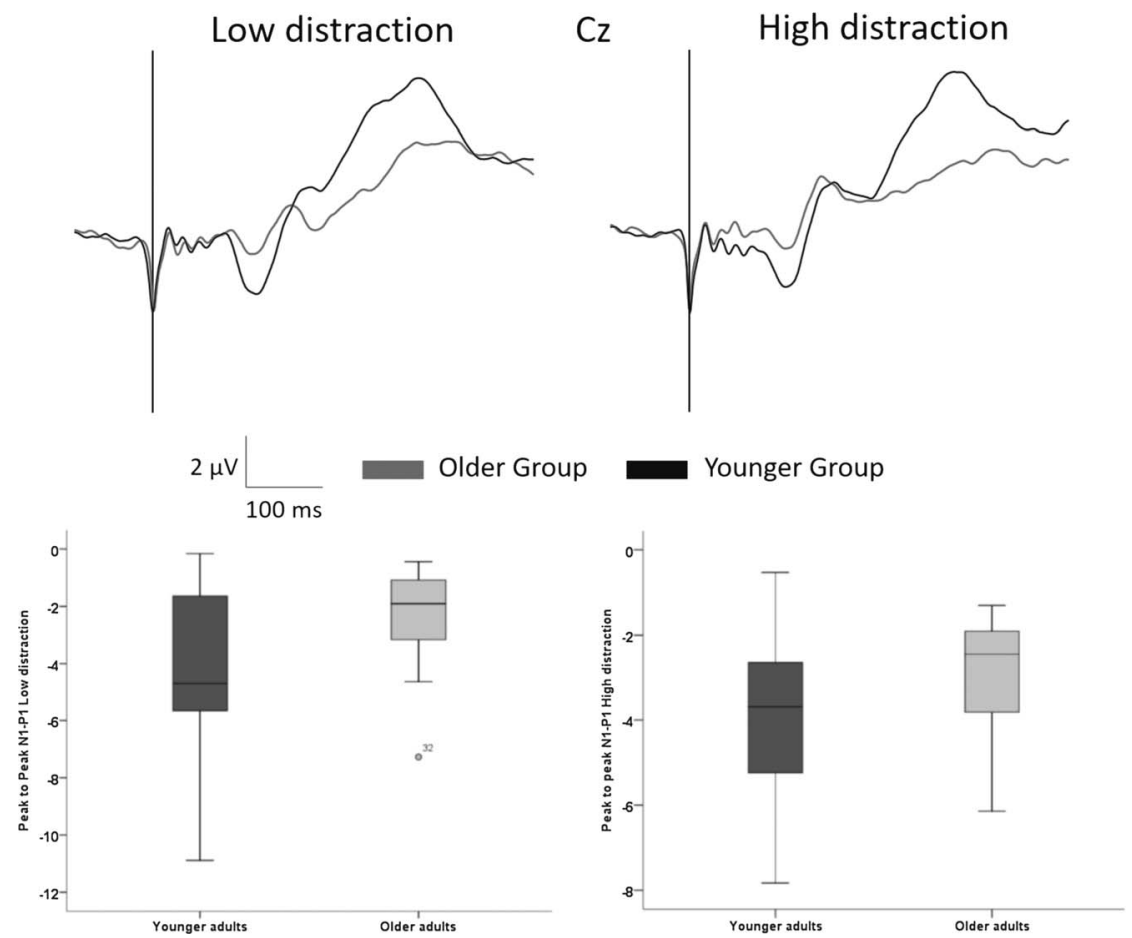

FIGURE 2. Pain-related evoked potentials (upper panel). Grand averages of pain-evoked potentials elicited by pain under the low- and high-distraction conditions at $\mathrm{Cz}$ electrode for younger (black lines) and older (gray lines) participants. Boxplot showing peak-to-peak N1-P1 amplitudes at Cz electrode for younger and older participants (lower panel). 
stimuli that are potentially significant for the integrity of the body $(12,13)$. In this regard, it is important to notice that similar reductions in evoked potentials have been found in older participants in response to stimuli of other sensory modalities (45-47). Indeed, in our study, older participants showed generalized reduced $\mathrm{P} 3$ potentials to visual letter stimuli (both target and no-target) of the distraction task, in comparison to younger ones (Supplemental Digital Content, http://links. lww.com/PSYMED/A688). Thus, reduced pain-evoked potentials in older adults may be mirroring a deficit in the activation of this general alarm/orienting system. This interpretation is also in line with psychophysiological research on pain, consistently showing that older participants display increased pain thresholds. Indeed, we also found that older participants showed increased pain thresholds in comparison to younger ones. In this regard, Lautenbacher et al. $(1,3)$ proposed that age might dull pain sensitivity, as it dulls vision and hearing, leading to a delayed detection of external threats (3). Our results seem to confirm this hypothesis based on EEG responses to pain, suggesting a reduction in the underlying processes involved in coding the salience of painful stimuli in the older population.

In contrast with this reduced brain response to pain, we found that older adults consistently reported more unpleasantness to pain than did younger participants. This result is in agreement with previous studies showing that older adults display reduced pain tolerance, making pain complaints become more likely (39). This increased affective response (which is still under debate; Lautenbancher et al. (1)), could reflect increased sensitivity to pain, a decrease in endogenous inhibitory capacity, deficient coping strategies, or age-related alterations in decision-making processes underlying pain assessment. In our paradigm, participants received three pain stimuli in each block of a 60 -second task and then rated the mean pain felt during the block. It is possible that the usual age-related decline in working memory and decision making $(48,49)$ affected pain assessment in older people (e.g., creating less reliable memory representations of pain stimuli). Further studies should deepen this question, but our results suggest that affective-motivational aspects of pain perception could be altered by aging.

\section{Limitations}

First, in our pain distraction paradigm, the painful stimuli were embedded within the cognitive tasks. Therefore, it is possible that the cognitive processes elicited by the tasks could have influenced the pain-evoked potentials. A similar issue has been discussed in the context of using other types of experimental tasks to modulate the level of attention directed to pain (counting or rating nociceptive stimuli, making arithmetic calculations, etc.; see Legrain et al. (11) for a review). However, it is important to note that both participant groups were exposed to the same level of task difficulty, as a result of our calibration procedure. Moreover, in both distraction conditions, participants executed responses to letters appearing at the same rate, only varying the cognitive load of the task. Therefore, it is very unlikely that pain-evoked potentials differences between groups were due to the concomitant cognitive task. Second, we did not find any significant correlations between pain ratings and pain-evoked potentials. However, this discrepancy has been found before by Özgül et al. (10), who showed that pain-evoked potential amplitudes decreased with age but were not related to subjective pain ratings. Furthermore, this lack of relationship between both measures is one of the main arguments supporting that the multimodal nature of the nociceptive-evoked potentials cannot be interpreted as a direct index of the subjective experience of pain (50). Third, older and younger participants differed in medication intake. However, hypertensive and antidepressant medication, which can modify pain thresholds (37), has been controlled as a covariate and resulted to be not significant. Fourth, the possible effect of fluctuating ovarian hormones during the menstrual cycle on pain perception over younger women (38) was not considered. Moreover, we cannot fully discard the possible effect of menopause in the described changes in pain perception due to aging in older women. Finally, our sample, although consistent with the typical sample size in ERP studies (51), was relatively small, which can lead to magnitude (i.e., size of the effect) and sign errors (i.e., direction of the effect) (52) when estimating differences between older and younger adults in pain perception. However, we have demonstrated an adequate internal consistency of the ERP responses.

\section{CONCLUSIONS}

Our results support the use of distraction as a therapeutic approach for older adults when pain intensity is moderate. Moreover, we also found that aging is characterized by reduced detection and coding of meaningful stimuli for the body, as shown by increased pain thresholds and attenuated pain-evoked potentials to painful electrical stimuli. This age-related reduction in the salience of pain is accompanied, paradoxically, by an increased subjective pain perception. We suggest that, because of executive function decline, older participants may have a reduced ability to create coherent perceptions and cope with painful events, leading to altered affective evaluation of pain stimuli. This phenomenon could be related to the increased vulnerability of older participants to develop chronic pain, as alterations in the affective components of pain perception are also commonly observed in chronic pain conditions $(53,54)$.

Source of Funding and Conflicts of Interest This study was supported by the Spanish Ministry of Economy, Industry and Competitiveness (PSI2016-78637-P AEI/FEDER-UE, PSI2017-88388-C4-1$R$ AEI/FEDER-UE, PSI2015-66295-R AEI/FEDER-UE), and the Spanish Ministry of Science and Innovation (PID2019-110096GBI00/ AEI /10.13039/501100011033). The authors declare no conflicts of interest.

\section{REFERENCES}

1. Lautenbacher S, Peters JH, Heesen M, Scheel J, Kunz M. Age changes in pain perception: a systematic-review and meta-analysis of age effects on pain and tolerance thresholds. Neurosci Biobehav Rev 2017;75:104-13.

2. Farrell MJ. Age-related changes in the structure and function of brain regions involved in pain processing. Pain Med 2012;13:S37-43.

3. Lautenbacher S. Experimental approaches in the study of pain in the elderly. Pain Med 2012;13:S44-50.

4. Marouf R, Caron S, Lussier M, Bherer L, Piché M, Rainville P. Reduced pain inhibition is associated with reduced cognitive inhibition in healthy aging. Pain 2014;155:494-502.

5. van der Meulen M, Kamping S, Anton F. The role of cognitive reappraisal in placebo analgesia: an fMRI study. Soc Cogn Affect Neurosci 2017;12:1128-37.

6. Wiech K. Deconstructing the sensation of pain: the influence of cognitive processes on pain perception. Science 2016;354:584-7.

7. Zhou S, Kemp J, Després O, Pebayle T, Dufour A. The association between inhibition and pain tolerance in the elderly: evidence from event-related potentials. Eur J Pain 2015; 19:669-76.

8. Zhou S, Després O, Pebayle T, Dufour A. Age-related decline in cognitive pain modulation induced by distraction: evidence from event-related potentials. J Pain 2015;16:862-72. 
9. Mueller D, Obermann M, Koeppen S, Kavuk I, Yoon MS, Sack F, Diener HC Kaube H, Katsarava Z. Electrically evoked nociceptive potentials for early detection of diabetic small-fiber neuropathy. Eur J Neurol 2010;17:834 41.

10. Özgül ÖS, Maier C, Enax-Krumova EK, Vollert J, Fischer M, Tegenthoff M Höffken O. High test-retest-reliability of pain-related evoked potentials (PREP) in healthy subjects. Neurosci Lett 2017;647:110-6.

11. Legrain V, Mancini F, Sambo CF, Torta DM, Ronga I, Valentini E. Cognitive aspects of nociception and pain. Bridging neurophysiology with cognitive psychology. Neurophysiol Clin 2012;42:325-36.

12. Torta DM, Legrain V, Mouraux A, Valentini E. Attention to pain! A neurocognitive perspective on attentional modulation of pain in neuroimaging studies. Cortex 2017;89:120-34.

13. Mouraux A, Iannetti GD. The search for pain biomarkers in the human brain Brain 2018;141:3290-307

14. Jurado MB, Rosselli M. The elusive nature of executive functions: a review of our current understanding. Neuropsychol Rev 2007;17:213-33.

15. Aschenbrenner AJ, Balota DA. Dynamic adjustments of attentional control in healthy aging. Psychol Aging 2017;32:1-15.

16. Lobo A, Saz P, Marcos G, Día JL, de la Cámara C, Ventura T, Morales Asín F, Fernando Pascual L, Montañés JA, Aznar S. Revalidation and standardization of the cognition mini-exam (first Spanish version of the Mini-Menta Status Examination) in the general geriatric population. Med Clin (Barc) 1999;112:767-74.

17. Kroenke K, Spitzer RL, Williams JB. The PHQ-9: validity of a brief depression severity measure. J Gen Intern Med 2001;16:606-13.

18. Garcia-Campayo J, Zamorano E, Ruiz MA, Pardo A, Perez-Paramo M, LopezGomez V, Freire O, Rejas J. Cultural adaptation into Spanish of the Generalized Anxiety Disorder-7 (GAD-7) scale as a screening tool. Health Qual Life Outcomes 2010;8:8

19. Sullivan MJL, Bishop SR, Pivik J. The Pain Catastrophizing Scale: development and validation. Psychol Assess 1995;7:524-32.

20. Oldfield RC. The assessment and analysis of handedness: the Edinburgh inventory. Neuropsychologia 1971;9:97-113.

21. Watson D, Clark LA, Tellegen A. Development and validation of brief measures of positive and negative affect: the PANAS scales. J Pers Soc Psychol 1988;54 $1063-70$.

22. Inui K, Tran TD, Qiu Y, Wang X, Hoshiyama M, Kakigi R. Pain-related magnetic fields evoked by intra-epidermal electrical stimulation in humans. Clin Neurophysiol 2002;113:298-304

23. Boivie J, Leijon G, Johansson I. Central post-stroke pain - a study of the mechanisms through analyses of the sensory abnormalities. Pain 1989;37:173-85.

24. Buhle J, Wager TD. Performance-dependent inhibition of pain by an executive working memory task. Pain 2010;149:19-26.

25. Zhang J, Mueller ST. A note on ROC analysis and non-parametric estimate of sensitivity. Psychometrika 2005;70:203-12.

26. Gratton G, Coles MG, Donchin E. A new method for off-line removal of ocular artifact. Electroencephalogr Clin Neurophysiol 1983;55:468-84

27. Oostenveld R, Fries P, Maris E, Schoffelen JM. FieldTrip: open source software for advanced analysis of MEG, EEG, and invasive electrophysiological data. Comput Intell Neurosci 2011;2011:156869.

28. Maris E, Oostenveld R. Nonparametric statistical testing of EEG- and MEG-data J Neurosci Methods 2007;164:177-90.

29. Legrain V, Bruyer R, Guérit J-M, Plaghki L. Nociceptive processing in the human brain of infrequent task-relevant and task-irrelevant noxious stimuli. A study with event-related potentials evoked by $\mathrm{CO}_{2}$ laser radiant heat stimuli. Pain 2003;103: 237-48.

30. Siedenberg R, Treede RD. Laser-evoked potentials: exogenous and endogenous components. Electroencephalogr Clin Neurophysiol 1996;100:240-9.

31. Legrain V, Guérit J-M, Bruyer R, Plaghki L. Attentional modulation of the nociceptive processing into the human brain: selective spatial attention, probability of stimulus occurrence, and target detection effects on laser evoked potentials. Pain 2002:99:21-39.

32. Kanda M, Fujiwara N, Xu X, Shindo K, Nagamine T, Ikeda A, Shibasaki H. Painrelated and cognitive components of somatosensory evoked potentials following $\mathrm{CO}_{2}$ laser stimulation in man. Electroencephalogr Clin Neurophysiol 1996;100: 105-14.

33. Lorenz J, Garcia-Larrea L. Contribution of attentional and cognitive factors to laser evoked brain potentials. Neurophysiol Clin 2003;33:293-301.

34. Thigpen NN, Kappenman ES, Keil A. Assessing the internal consistency of the event-related potential: an example analysis. Psychophysiology 2017;54:123-38.

35. Boudewyn MA, Luck SJ, Farrens JL, Kappenman ES. How many trials does it take to get a significant ERP effect? It depends. Psychophysiology 2018:55:e13049.

36. Clayson PE, Miller GA. ERP Reliability Analysis (ERA) Toolbox: an opensource toolbox for analyzing the reliability of event-related brain potentials. Int J Psychophysiol 2017;111:68-79.

37. Saccò M, Meschi M, Regolisti G, Detrenis S, Bianchi L, Bertorelli M, Pioli S, Magnano A, Spagnoli F, Giuri PG, Fiaccadori E, Caiazza A. The relationship between blood pressure and pain. J Clin Hypertens (Greenwich) 2013;15:600-5.

38. Michaelides A, Zis P. Depression, anxiety and acute pain: links and management challenges. Postgrad Med 2019;131:438-44.

39. Sassenhagen J, Draschkow D. Cluster-based permutation tests of MEG/EEG data do not establish significance of effect latency or location. Psychophysiology 2019;56:e13335.

40. Ebert MH, Kerns RD, editors. Behavioral and Psychopharmacologic Pain Management. Cambridge, UK: Cambridge University Press; 2010.

41. Creac'H C, Bertholon A, Convers P, Garcia-Larrea L, Peyron R. Effects of aging on laser evoked potentials. Muscle Nerve 2015;51:736-42.

42. Gibson SJ, Farrell M. A review of age differences in the neurophysiology of nociception and the perceptual experience of pain. Clin J Pain 2004;20:227-39.

43. Gibson SJ, Voukelatos X, Ames D, Flicker L, Helme RD. An examination of pain perception and cerebral event-related potentials following carbon dioxide laser stimulation in patients with Alzheimer's disease and age-matched control volunteers. Pain Res Manag 2001;6:126-32.

44. Chao CC, Hsieh ST, Chiu MJ, Tseng MT, Chang YC. Effects of aging on contact heat-evoked potentials: the physiological assessment of thermal perception. Muscle Nerve 2007;36:30-8.

45. Terrasa JL, Montoya P, González-Roldán AM, Sitges C. Corrigendum: Inhibitory control impairment on somatosensory gating due to aging: an event-related potential study. Front Hum Neurosci 2018;12:280.

46. Wickremaratchi MM, Llewelyn JG. Effects of ageing on touch. Postgrad Med J 2006;82:301-4

47. Shaffer SW, Harrison AL. Aging of the somatosensory system: a translational perspective. Phys Ther 2007;87:193-207.

48. Kirova AM, Bays RB, Lagalwar S. Working memory and executive function decline across normal aging, mild cognitive impairment, and Alzheimer's disease. Biomed Res Int 2015;2015:748212.

49. Löckenhoff CE. Aging and decision-making: a conceptual framework for future research — a mini-review. Gerontology 2018;64:140-8.

50. Iannetti GD, Hughes NP, Lee MC, Mouraux A. Determinants of laser-evoked EEG responses: pain perception or stimulus saliency? J Neurophysiol 2008;100:815-28.

51. Clayson PE, Carbine KA, Baldwin SA, Larson MJ. Methodological reporting behavior, sample sizes, and statistical power in studies of event-related potentials: barriers to reproducibility and replicability. Psychophysiology 2019;56:e13437.

52. Gelman A, Carlin J. Beyond power calculations: assessing type S (sign) and type M (magnitude) errors. Perspect Psychol Sci 2014;9:641-51.

53. Borsook D, Edwards R, Elman I, Becerra L, Levine J. Pain and analgesia: the value of salience circuits. Prog Neurobiol 2013;104:93-105.

54. Malfliet A, Coppieters I, Van Wilgen P, Kregel J, De Pauw R, Dolphens M, Ickmans $\mathrm{K}$. Brain changes associated with cognitive and emotional factors in chronic pain: a systematic review. Eur J Pain 2017;21:769-86. 